\title{
Model-to-Model Transformations for Efficient Time-domain Verification of Concurrent Models by NuSMV Modules
}

\author{
Miguel Carrillo ${ }^{1}{ }^{\mathrm{a}}$, Vladimir Estivill-Castro ${ }^{2} \mathbb{1}^{\mathrm{b}}$ and David A. Rosenblueth ${ }^{1} \mathbb{C}^{\mathrm{c}}$ \\ ${ }^{1}$ Instituto de Investigaciones en Matemáticas Aplicadas y en Sistemas, Universidad Nacional Autónoma de Mexico, \\ Apdo. 20-126, 01000 Mexico D.F., Mexico \\ ${ }^{2}$ School of Information and Communication Technology, Griffith University, Brisbane 4111, Queensland, Australia \\ miguel.mcb@gmail.com,v.estivill-castro@griffith.edu.au,drosenbl@unam.mx
}

Keywords: Model Checking, Kripke Structure, Value-domain Verification, Time-domain Verification.

Abstract: We introduce and describe an algorithmic transformation from the formalism of arrangements of logic-labelled finite-state machines (LLFSMs) into NuSMV modules (and its implementation as a model-to-model ATL transformation from an Ecore meta-model to the NuSMV language). Our transformation benefits from using modules and integers of NuSMV to improve the efficiency in the construction and verification of the model. Moreover, we can handle predicates about time. Thus, we enable verification of LLFSMs in the time domain. Our transformation is a considerable improvement in efficiency. Compared with earlier transformation algorithms developed by us, the one presented here produces concise NuSMV files (in an example, 130,295 lines were reduced to 418). We thus show that it is possible to automatically translate arrangements of LLFSMs to concise models that can be efficiently and formally verified.

\section{INTRODUCTION}

We are concerned with model-driven software development where the specification of a system is executable. In practice, it does not suffice to have an executable specification, as such a specification may also contain errors. Although verifying non-executable models brings insights into the correctness of the implementation, errors can be introduced in the simplification of the executable program to a model, or the implementation of a non-executable model into code. Our objective is to show, within model-driven software development, that it is possible to automatically translate arrangements of LLFSMs to models that can be efficiently verified with model checking.

We use logic-labelled finite-state machines (LLFSMs) to model and specify the behaviour of systems. Earlier algorithms processing LLFSMs for modelchecking required the explicit construction (McColl and Estivill-Castro, 2017; Estivill-Castro and Rosenblueth, 2011; Estivill-Castro and Hexel, 2013) of the corresponding Kripke structure (Seshia et al., 2018, Section 3.5, Definition 2). Our first contribution in this paper is the application of model-to-model

\footnotetext{
a (iD https://orcid.org/0000-0003-2105-3075

b (iD https://orcid.org/0000-0001-7775-0780

c (i) https://orcid.org/0000-0001-8933-8267
}

transformations from an Ecore ${ }^{1}$ meta-model to SMV modules (Cimatti et al., 2000). Because we use the SMV modules of $\mathrm{NuSMV}^{2}$, we obtain a tremendous improvement in the efficiency of the description of the verifiable model. For instance, for the four LLFSMs of a microwave oven example, our NuSMV model is only 418 lines long. The two separate implementations of our early algorithms, by contrast, deliver NuSMV files for the same problem that have 130,295 lines one, and 110,567 lines the other. Work with the earlier algorithms reports similar figures (McColl and Estivill-Castro, 2017). These earlier algorithms suffer also from not being able to use the native integers in NuSMV (Cimatti et al., 2000). Such attempts, however, proved the first correctness results regarding no overflow of the timer control for such an example. In Section 3.4, we will show that the size of the output of our algorithm is linear in the number of states of

\footnotetext{
${ }^{1}$ Ecore is the first piece of the Eclipse Modeling Framework for describing software models and meta-models.

${ }^{2}$ In NuSMV's language for describing Kripke structures, modules are the fundamental tool for composability; default composition of modules in NuSMV is synchronous (asynchronous composition through processes is deprecated), where a single step of the resulting Kripke structure corresponds to a simultaneous step of all component modules. We will use "NuSMV" for the model-checker and "SMV" for its input language.
} 
the arrangement of LLFSMs.

Models with LLFSM are verifiable for valuedomain properties. Few properties can be verified in the time domain, but only as bounded model checking, that is, considering only a finite prefix of a path that may be a solution to an existential modelchecking problem (Biere et al., 1999). Our second contribution is to explicitly transform the behaviour of an arrangement of LLFSMs using time predicates into Kripke structures that includes explicitly timers as modules. Thus, time-domain properties that explicitly mention time units are verifiable with a standard model checker, such as NuSMV (Cimatti et al., 2000).

\section{EVENTS VS BOOLEAN EXPRESSIONS}

Perhaps the most prominent modelling language for model-driven software development is the Unified Modeling Language (UML). In UML, important abstractions for behaviour are UML statecharts. UML statecharts label transitions between states with events. This is a common feature to state-based systems derived from Harel's seminal introduction of STATEMATE (Harel et al., 1990). Modelling tools, such as OMT, were influenced by STATEMATE and adopted the event-driven approach (Rumbaugh et al., 1991). Executable state diagrams and tools from ROOM (Selic et al., 1994) also became event-driven. Event-driven semantics was disseminated with executable state machines by QM (Samek, 2008). International standards such as the Specification and Description Language (SDL) for telecommunications systems use event-driven communicating extended finite-state machines for modelling behaviour (ITUT Study Group 17, 2002). Even tools for multiagent systems simulation, such as repast (Ozik et al., 2015), adopt the event-driven view.

Modelling with event-driven statecharts has several advantages, but many authors have already discussed important drawbacks (Mellor, 2000; von der Beeck, 1994). A first drawback with event-driven statecharts is the ambiguous loose-ends of the Run Until Completion semantics. Although apparently simple in that all new events are queued while handling the current event, this already raises two alternatives for events caused by the current event. Such derived events could either go at the end of the queue (if they are considered new events) or could be at the front of the queue (if they are considered causes of the new event). In either case, if there is more than one listener for the same derived event, then we break the assumption that no two events happen at the same time. Further drawbacks are the evaluation of expressions or reading of variables. For example, should the guard associated with an event that labels a transition be evaluated when the event happens or when the event is de-queued? The first option implies running code that interrupts the current event and violates the Run Until Completion semantics, but evaluating the guard much later than when an event occurred may be completely inadequate for the intended behaviour. Because of the mental load that this semantics implies, UML scenarios using it are hard for humans to resolve (Estivill-Castro and Hexel, 2019). Moreover, UML's variation points on event handling may result in several alternative semantics for UML executable models. (Besnard et al. (2018) report that the verification of a (value-domain) property results in an error when using FifoEventPool, but is successful when using OrderedList-DeferredEventPool.)

UML is effective in the design of systems where the environment is unlikely to produce a shower of events. Graphical User Interfaces (GUIs) are a good example of the success of event-driven systems. The reason is that a GUI user is unlikely to click in two widgets without time gaps in between. However, this is not the case for Cyber-physical systems, where showers of events are common. Queues are a major problem for event-driven system and fundamental theorems have long-time existed regarding the impossibility of confirming claims in the time domain or for real-time systems (Lamport, 1984). Verification of event-driven systems leads directly to the stateexplosion that typically blocks model checking because all possible permutations of the arrival of events and their progress in queues need to be considered.

An alternative are Logic-Labelled Finite-State Machines (for short LLFSMs, but also known as procedural state machines (Drusinsky, 2006, Page 51)). LLFSMs enable concurrency under a prescribed schedule: "because [the automaton] can access the input symbols at any time, it can visit states as fast as we wish" (Drusinsky, 2006, Page 15). These behavioural models label transitions with Boolean expressions (or predicates also called enabling functions (Cheng and Krishnakumar, 1993)) and have a long trajectory in verification (Devadas et al., 1991). LLFSMs can be used beyond the realms of reactive systems as the transitions can be queries to an expert/deliberative system that determines whether the transition should trigger or not (Estivill-Castro et al., 2016). They have been used to formally verify more efficiently and more effectively more properties than other methods in the following examples: a microwave oven, a mine pump, a power car-window, and an ambulatory pump. 


\subsection{Related Work}

The deployment of critical fault-tolerant real-time systems has had a sustained interest in linking models to verification (Poledna, 1996). In particular, for model-driven software development, where models are considered the primary artefacts of the software, model checking becomes of the utmost importance. For example, for the development of safety-critical avionics software, there has been constant interest in (Meenakshi et al., 2006) 1) tools that automatically translate certain Simulink models into input language of a suitable model checker, and 2) ensuring that the size of the translated model is not excessive for verification. However, we emphasize that our goal is to reduce what others have identified as the semantic gap between models and code (Besnard et al., 2018). The issue is that most model-checking activity verifies a model that abstracts away some aspects of the running software (Besnard et al., 2018). Such aspects are usually also relevant when design models are converted into executable code. Moreover, diagnosis processes are complicated since separate (sometimes even manual) transformations into a formal language are performed to enable formal verification (Besnard et al., 2018). A long list of tools has been reviewed and each displays such semantic gap or diagnosis difficulty to some extent (Besnard et al., 2018, Section 7).

We illustrate the aspects of this gap by reviewing STP (Clarke and Heinle, 2000), an approach for converting STATEMATE statecharts into SMV. This revision will also highlight our preference for LLFSMs. First, despite attempting to handle the fundamental composition notation of STATEMATE (that is, hierarchical nesting), STP cannot handle inter-level transtions (Clarke and Heinle, 2000; Bhaduri and Ramesh, 2004). Remarkably, events are converted to event-variables that have the value true for exactly one SMV time step (that is, the translation is from LLFSMs instead of STATEMATE) and compound events (the events derived from an event) are treated as plain Boolean variables. While model checking will provide some insights about visiting states and transitions, there is not action language. In contrast with our modelling, moreover, there is no notion of time. After one event, nothing can happen (no new event, and no input can change), until the model is stable (Clarke and Heinle, 2000; Bhaduri and Ramesh, 2004). Thus, the verifications correspond to a mathematical model that is not implementable. The semantics assumes that event handling completes ahead of any other event and event ordering is guaranteed by the environment. Also the modelling assumes the environment behaves friendly and all non-determinism is resolved by closing the system to a suitable choice by the environment (user).

A review (Bhaduri and Ramesh, 2004) of several other similar translations (to SMV or SPIN) shows that a crucial issue in formal verification of statecharts is their semantics (in some cases, for instance RSML, the translation does not preserve the semantics). Most of those translations reviewed suffer from the same issues as STP: they eliminate events, treating them as Boolean variables (and returning to LLFSMs), eliminating all need to model the queueing of events, and leaving aside timing issues. Most of them support closed systems only (Bhaduri and Ramesh, 2004). If the method/tool attempts to queue events, it is a translation to SPIN that suffers from other limitations (only useful for deadlock detection, or only one statechart).

\section{FROM ARRANGEMENTS OF LLFSMS TO EFFICIENT MODULES}

We now provide an algorithm transforming an arrangement of LLFSM into a set of SMV modules. We first describe the tools supporting our argument that such an algorithm produces a description of the verifiable model that is significantly more efficient than that of earlier work. Next, we will discuss the obstacles in mapping each LLFSM to a module in SMV. We will reduce our problem to that of translating LLFSMs that only have blocks of code in their OnEntry sections (equivalently, they have no sections) and where blocks of code in a state have no dependencies. We follow this by the description of our algorithm to transform LLFSMs where no transition has an AFTER predicate.

\subsection{The Tools and Implementations}

In model checking, systems are categorised as open or closed (Seshia et al., 2018, Page 88). Closed systems have no inputs, and are frequently those that are formally verified. When dealing with an open system, a corresponding closed system is used by composing the open system with a model of its environment. In particular, if the open system were to receive an input supplied by a user, we adopt the convention (and thus modelling the user) that the value of the input variable is any non-deterministic value in its domain. Thus, the corresponding Kripke structure has, on a state reading an input, as many successor states as possible values the input could receive. The Kripke structure is then non-deterministic and mod- 
els the fact that the environment can pick the value arbitrarily without the control of the software.

An important property of LLFSMs is that they are executable. Moreover, they are not only interpretable, but can be compiled. The clfsm scheduler, available for downloading, ${ }^{3}$ enables extended LLFSMs where the action language is $\mathrm{C}++$. We, however, will use a subset of IMP (Winskel, 1993) as the action language, a simple imperative language, because the main purpose of this paper is to demonstrate the transformations of the imperative statements (and not of elaborate data structures or objects). We will use IMP's arithmetic expressions and Boolean expressions. However, from IMP's commands we take the empty command, the assignment and sequencing. What we exclude from IMP are two control commands: the selection (if-then-else) and the iteration (while-do). The reason is that both these constructs can be emulated by LLFSMs. Thus, by the structured program theorem (also called the BoehmJacopini theorem), LLFSMs are Turing complete.

We support this paper with the release of a LISP interpreter of arrangements of LLFSMs. This demonstrates that all models are executable. This interpreter enables to run arrangements of LLFSMs where interaction reveals the open nature of the system. Moreover, this LISP interpreter has an option -k so it can produce the corresponding closed Kripke structure using the methods mentioned in the introduction, where all states and transitions of the state system are listed. Thus, this option produces the closed system for verification. In particular, its output is readable by NuSMV (Cimatti et al., 2000) and then one appends the corresponding value-domain CTL or LTL formulas to verify the closed but non-deterministic model. This confirms that LLFSMs are both executable and verifiable in the value domain. We additionally provide the following files.

1. The corresponding Ecore files that define the meta-models discussed in this paper.

2. xmi files of examples of arrangements of LLFSMs (including the Microwave (Shlaer and Mellor, 1992; Myers and Dromey, 2009) and the Level-Crossing (Besnard et al., 2018)).

3. ATLAS Transformation Language (ATL) programs that implement the transformations described in this paper.

All are downloadable from earlier site.

\footnotetext{
${ }^{3}$ http://mipal.net.au/downloads.php.
}

\subsection{LLFSMs with no Sections}

The generic conversion of an LLFSMs model to SMV modules requires that we determine precisely the syntax and the semantics of LLFSMs. Therefore, besides the implementations mentioned earlier, we present here detailed definitions of LLFSMs. Figure 1 presents the Ecore meta-model for LLFSMs. We will start the description of our transformation by mentioning two aspects where LLFSMs are analogous to UML's statecharts, and thus, adoption of LLFSMs is accessible to UML users. First, we have already mentioned that in LLFSMs, each transition is labelled by a Boolean expression, and not an event. Thus, users familiar with UML will find that LLFSMs transitions are labelled only by guards. Because transitions are not labelled by events, the transitions out of a state $S$ are structured as a sequence (they are not just a set; they have an order). The order of the transitions out of $S$ is the order of evaluation of the corresponding expressions. In particular, the second transition can only fire if the guard of the first transition is false and its own transition label evaluates to true. In general, the guard of the $i$-th transition is $\neg g_{1} \wedge \cdots \wedge \neg g_{i-1} \wedge g_{i}$, where $g_{j}$ is the guard of the $j$-th transition. It is important to observe that the behaviour designer does not need to explicitly list this potentially long Boolean expression. However, when translating such a guard to a SMV module, the guard must be explicitly defined.

Second, a state in LLFSMs has three sections analogous to the states of UML's statecharts, but LLFSMs' semantics is more precise. The OnEntry section corresponds to code that is executed only the first time the thread of execution arrives at the state (from another state). The OnExit section is executed when a transition fires (its Boolean expression evaluates to true), prior to any code in the target state. The Internal section is evaluated only when the sequence of transitions out of the state has been exhausted with none of them evaluating to true. These sections can be considered syntactic sugar for state transition systems that have no sections in their states. Nevertheless, Figure 2 illustrates the non-trivial translation we perform from LLFSMs with states that do have sections, to LLFSMs with states that do not have sections. This also needs to be made explicit, since the NuSMV model checker needs to be explicitly alerted of the intermediate states of execution implied by the sections of states. Figure 2 illustrates a state with sections, two self transitions and two other transitions. The sections imply that after the execution of the OnEntry, there is a new state of execution for the other sections, so that there is an asymmetry with the OnExit (Estivill- 


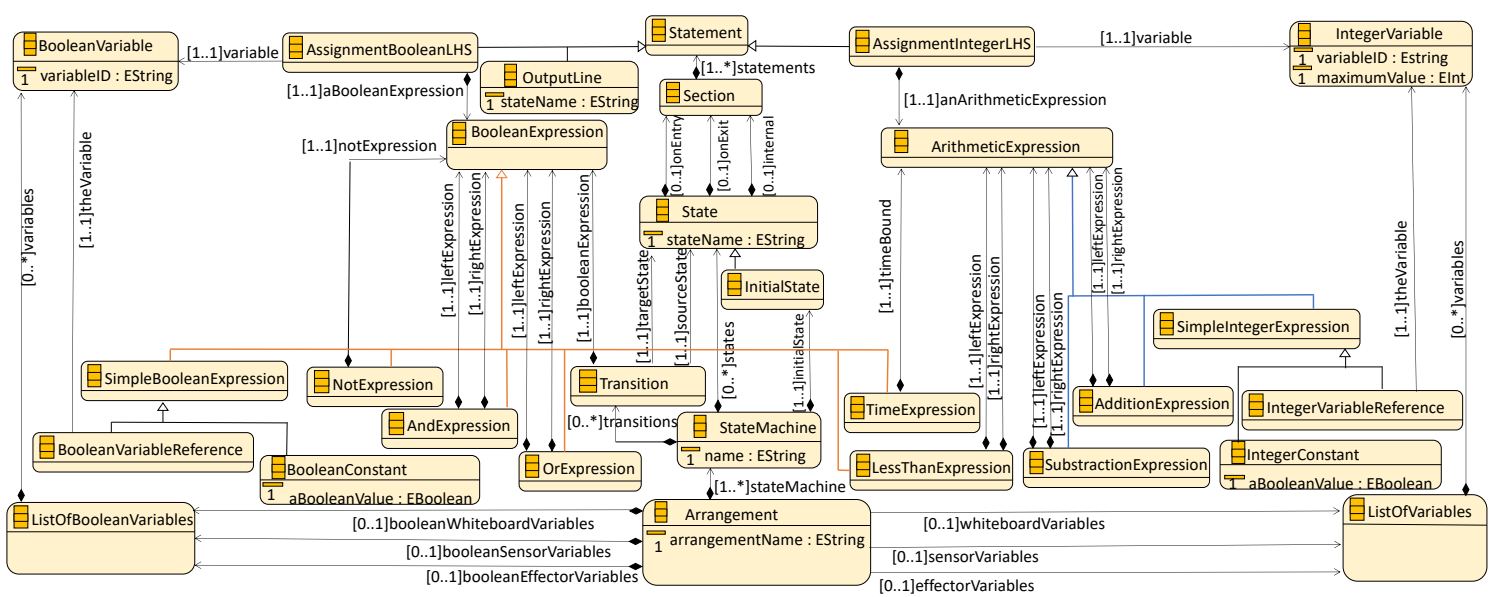

Figure 1: The meta-model that defines the behaviour models as an arrangement of LLFSMs for IMP.

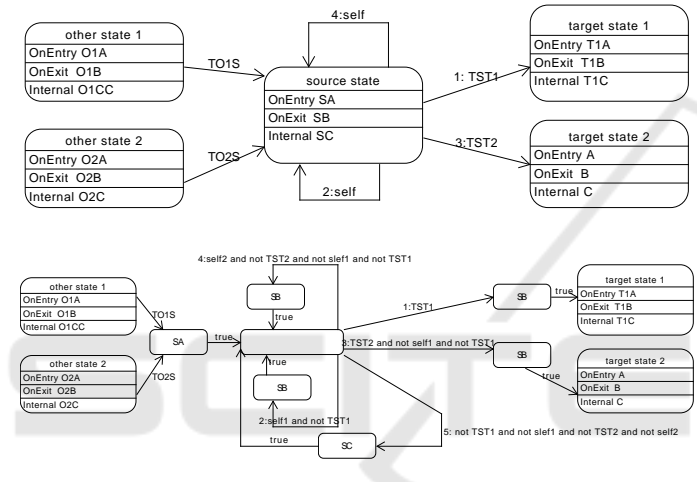

Figure 2: Illustration of the transformation that builds states without sections from states with sections.

Castro and Hexel, 2019). And while OnExit code is executed only on arrival from another state, self transitions do execute the OnExit, skip the Internal, and ensure the state is not terminal (in LLFSMs, states without exiting transitions are terminal). In what follows, we can assume that our models of behaviour are LLFSMs with only actions in the OnEntry sections.

Along the same lines, is the translation of executable blocks in LLFSMs. LLFSMs are Соттиnicating Extended State Machines (Kang and Lee, 1993). They are extended because LLFSMs use variables in (assignment) statements in the sections of states (and in the Boolean expression labelling the transitions). Theoretically, the use of variables (from an action language) is a technicality to avoid a large number of states; but since our target SMV can manipulate integer and Boolean variables, we will use variables of these two types.

LLFSMs are communicating because the variables can be shared between state machines of an arrangement. The shared variables are called whiteboard variables or external variables. Variables local to a finite state machine are not shared.

Blocks of code in IMP (Winskel, 1993) are sequences of assignments of the form

$$
\langle\text { variable }\rangle::=\langle\text { expression }\rangle \text {. }
$$

The assignments are typed. Thus, if the left-hand side (LHS) is a Boolean variable, the right-hand side (RHS) is a Boolean expression, and alternatively, if the LHS is an integer variable, then the RHS is an integer expression.

These blocks of code represent the next hurdle for translating to SMV modules. If the sequence of assignments do not represent a dependency that causes an intermediate state of computation, then the translation is direct. For instance, if the LHS of each assignment never appears in a later assignment, then the sequence of assignments in a block translates directly one to one to SMV code:

$\begin{array}{cccc}v_{1} & ::= & e_{1} \\ v_{2} & ::= & e_{2} \\ \vdots & & \vdots \\ v_{t} & ::= & e_{t}\end{array} \rightarrow \begin{array}{cccc}\operatorname{next}\left(v_{1}\right) & = & e_{1}^{\prime} \\ \operatorname{next}\left(v_{2}\right) & = & e_{2}^{\prime} \\ \vdots & & \vdots \\ \operatorname{next}\left(v_{t}\right) & = & e_{t}^{\prime}\end{array}$

where $e_{i}^{\prime}$ is the translation of $e_{i}$ from expression in IMP (and their operators) to expression in SMV.

If, however, the sequence of assignments displays a dependency, in particular a variable on the LHS appears in a later assignment on the RHS or the LHS, we must perform a transformation that eliminates the implicit sub-state of IMP. Algorithm 1 is the pseudocode of our ATL implementation, which uses a dictionary of pairs (variable,latest-SMV-expression). The dictionary keeps a history of assignments. For instance, if we the first assignment is $x:=y+1$, the dictionary has the pair $\langle(\mathrm{x},(\mathrm{y}+1))\rangle$. If the next assignment is $z:=x+3$, the dictionary is updated. First, free variables in expressions are replaced by expressions in the current dictionary, and second the dic- 


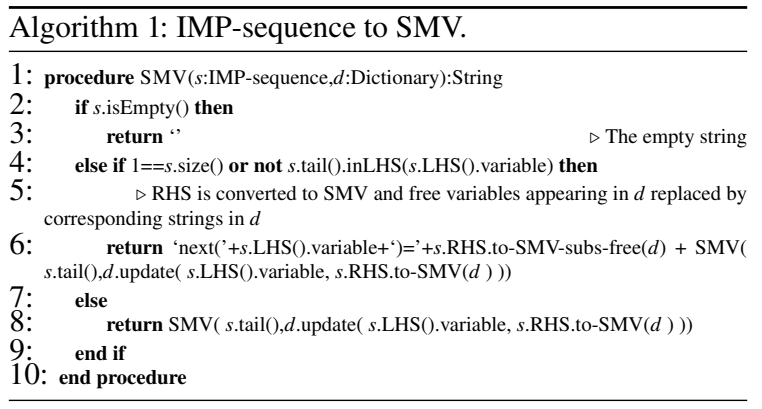

tionary updates its entries. Thus, the new dictionary is $\langle(\mathrm{x},(\mathrm{y}+1)),(\mathrm{z},((\mathrm{y}+1)+3))\rangle$.

When the sequence $s$ of IMP assignments is not empty and of length 1 , the translation to SMV is a next clause of the corresponding variable on the LHS, and the SVM result of binding the free variables on the RHS using the dictionary (and replacing operators of IMP to SMV).

When the sequence $s$ of IMP assignments is longer than 1 , we analyse the first assignment. If the corresponding variable on the LHS appears later again on the LHS, there is no output because we cannot have two SMV-statements of the form "next $(v)=$ " for the same variable $v$. Only the last one will produce output. We always process the tail of $s$ recursively with an updated dictionary. The dictionary accumulates the expressions, and records all over-writes. In our running example, if the third assignment is $z:=y+$ $x$, the second entry in the dictionary is updated. Now the dictionary is $\langle(\mathrm{x},(\mathrm{y}+1)),(\mathrm{z},(\mathrm{y}+(\mathrm{y}+1)))\rangle$.

An arrangement of LLFSMs is executed in a single tread. The individual finite-state machines are executed concurrently, and in a predefined schedule. As we already mentioned, this reduces the uncontrolled concurrency of event-driven systems, and a significant part of the state explosion for model checking. In what follows, we assume that LLFSMs hold executable code only in their OnEntry section and that code does not introduce sub-Kripke states (the assignments of that code can be treated atomically).

The transformations steps described so far may seem straightforward; however, they are far from immediate when implementing them with ATL.The first difficulty is the order in which to apply the transformations so that they are applied to every state (in the case of flattening each state to having code on the OnEntry section) and to every block (sequence of assignments) in such a way that there are no intermediate computational states. The second complexity is that these transformations demand contextual information. They cannot be applied to the state (or the block of code) without knowledge of all the transitions arriving and departing the corresponding state, the expressions labelling those transitions, and the scope of the variables involved.

This brings us to the point in our transformation of how variables in the LLFSM correspond to variables in the SMV modules we produce. While each LLFSM will be translated into an SMV module, we assign what we refer to as an owner module to each variable (those that provide the capability to use integers and Booleans and provided the adjective extended mentioned earlier). In the resulting set of modules, we declare each variable with an SMV declaration in the module that owns it.

Whether the variable is external or local, we assign an SMV module as its owner. The assignments do not require human intervention.

1. If a variable $v$ is local to an $\operatorname{LLFSM} M$, it has the module for $M$ as $v$ 's owner.

2. If a variable $v$ is external, and does not appear on the LHS of any assignment, $v$ is considered as belonging to the environment (an input or a sensor) and the SMV model is closed, as discussed before (but the variable $v$ can non-deterministically adopt any value in its domain at each Kripke state). Such a variable $v$ has as its owner an SMV module named main (which would be also used for the predefined schedule of the arrangement).

3. If a variable is external and appears on the LHS of assignments but of only one LLFSMs, then we assign the SMV module for $M$ as the owner.

4. If a variable $v$ appears on the LHS of assignments in states of two or more LLFSMs, the SMV module for $v$ is the main module and those modules having it (either on their LHS or RHS) receive it as a parameter by reference.

This last point of the transformation may seem contentions as, at first glance, an apparent race condition could present itself if two or more LLFSMs in the arrangement that write into a variable (they have it in the LHS of some of their assignments) were to execute in parallel. Recall that SMV composes modules under synchronous composability. However, we will ensure that the semantics of LLFSMs (that a predefined schedule, typically one turn for each LLFSM in the arrangement) is enforced by the module main. Thus, we preserve that only one LLFSMs executes a ringlet (Estivill-Castro and Rosenblueth, 2011) in its current state and only one LLFSM holds the current turn. We stress that there is only one thread per arrangement.

We deal now with the communicating feature of LLFSMs. LLFSMs may read the values of external variables on the RHS of their assignments. We achieve this by identifying, for each LLFSM $M$, all 
the variables on the RHS of all its assignments (in all its states) of which it is not the owner. This set of variables, plus those from Case 4 above becomes a list of formal parameters to the SMV module for $M$.

With these preliminaries, we can now complete the description of our algorithm for producing a set of SMV modules that corresponds to the behaviour defined by an arrangement of LLFSMs. The merits of the transformation are that it is (a) linear in the size of the description of the arrangement and (b) completely independent of the states of computation of the arrangement (as opposed to our previous approaches).

\subsection{Translating LLFSMs with no AFTER}

We present our algorithm as a series of transformation steps which are analogous to ATL's declarative rules. However, for the purposes of illustrating this presentation, we introduce a simple arrangement of two LLFSMs (refer to Figure 3, which is drawn by our ATL program transforming LLFSMs arrangements into dot (Gansner et al., 2015) input).

An arrangement of LLFSMs with actions only in the OnEntry section (such as the example in Figure 3) can be thought of as an array of Christmas lights, where only one arrow fires at each point in time, because there is a schedule (a pattern) that programs the display. Figure 3 shows each LLFSM in a rectangle. The LLFSMs scheduler assigns a turn to the arrangement in round-robin fashion.

Transformation Step: Our SMV model will consist of a module for each of LLFSM (total $n$ ) and a main module with a variable turn. The domain of turn is from 0 to $n-1$.

Illustration: The code below is what our ATL transformation produces for Figure 3, except that we have here added the initialisation turn $=0$ (Line 6) to the first LLFSM in the arrangement (but we typically verify properties for any possible order of the LLFSMs in the arrangement).

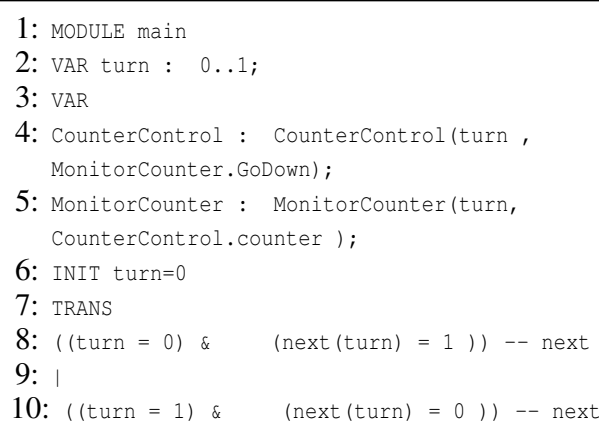

Transformation Step: A module instantiation for each LLFSM in the arrangement is listed in module main with actual parameters identified with the prefix of the owner (or no prefix, if main is the owner).

Illustration: The earlier code includes the declaration of the two modules (Line 4 and Line 5) corresponding to the two LLFSMs in Figure 3. It also shows the actual parameters. Both modules will read the value of turn.

Transformation Step: The module main schedules the variable turn so that the synchronous composition of modules by SMV results in only one module advancing a step. The round-robin schedule is a series of conditions

$$
(\operatorname{turn}=i) \&(\operatorname{next}(\operatorname{turn})=i \bmod n)
$$

for $i=0, \ldots, n-2$ and for $i=n-1$

$$
(\operatorname{turn}=i) \&(\operatorname{next}(\operatorname{turn})=0) .
$$

In general, for LLFSM $M_{i}$, all the transitions in the SMV module will have the test $(\operatorname{turn}=i$ ). Therefore, no transition fires in the SMV model, except for a module whose turn is next, and main performs the round-robin assignment of the turn.

Transformation Step: Not only LLFSMs are numbered to be identified. The states within a LLFSM are also numbered. Transitions are numbered sequentially within a pair $(i, j)$ consisting of their machine number $i$ and their transition number $j$ (and respecting their order when they share a source state).

Illustration: For example, state LetsGoUp is State 1 in Machine 1 (MonitorCounter), while state Decrement is State 4 in Machine 0 (refer to Figure 3). So in Machine 0 (CounterControl), the transition from Start to Test, the transition is Tid=01.

Transformation Step: Each machine $M_{i}$ will have a program counter (pc) indicating its current state, with values from 0 to the number of states in $M_{i}$. For a transition Tid= $i j$ with label $b e_{j}$ to fire, it must be that it is the $i$-th machine's turn, the program counter is in the source state $s$ of Tid= $i j$, the labelling expression $b e_{j}$ evaluates to true, and all other transitions with the same source state as source do not fire (remember that there is an order for transitions out of the same state). For each transition Tid $=i j$ with label $b e_{j}$, we define conditions (in SMV)

$$
\operatorname{condT}_{i j}:=(\operatorname{turn}=i) \&(\operatorname{pci} i=s) \quad \& b e_{j} .
$$

When a transition Tid=ij fires, it has the consequence of advancing the Kripke structure to the next Kripke state with a new valuation. The first consequence is the update of the program counter (the machine changes state). The other consequence is the effects of the block of assignment statements in the OnEntry of Tid=ij's target state. We emphasise one relevant point: because of the semantics of SMV, for those 


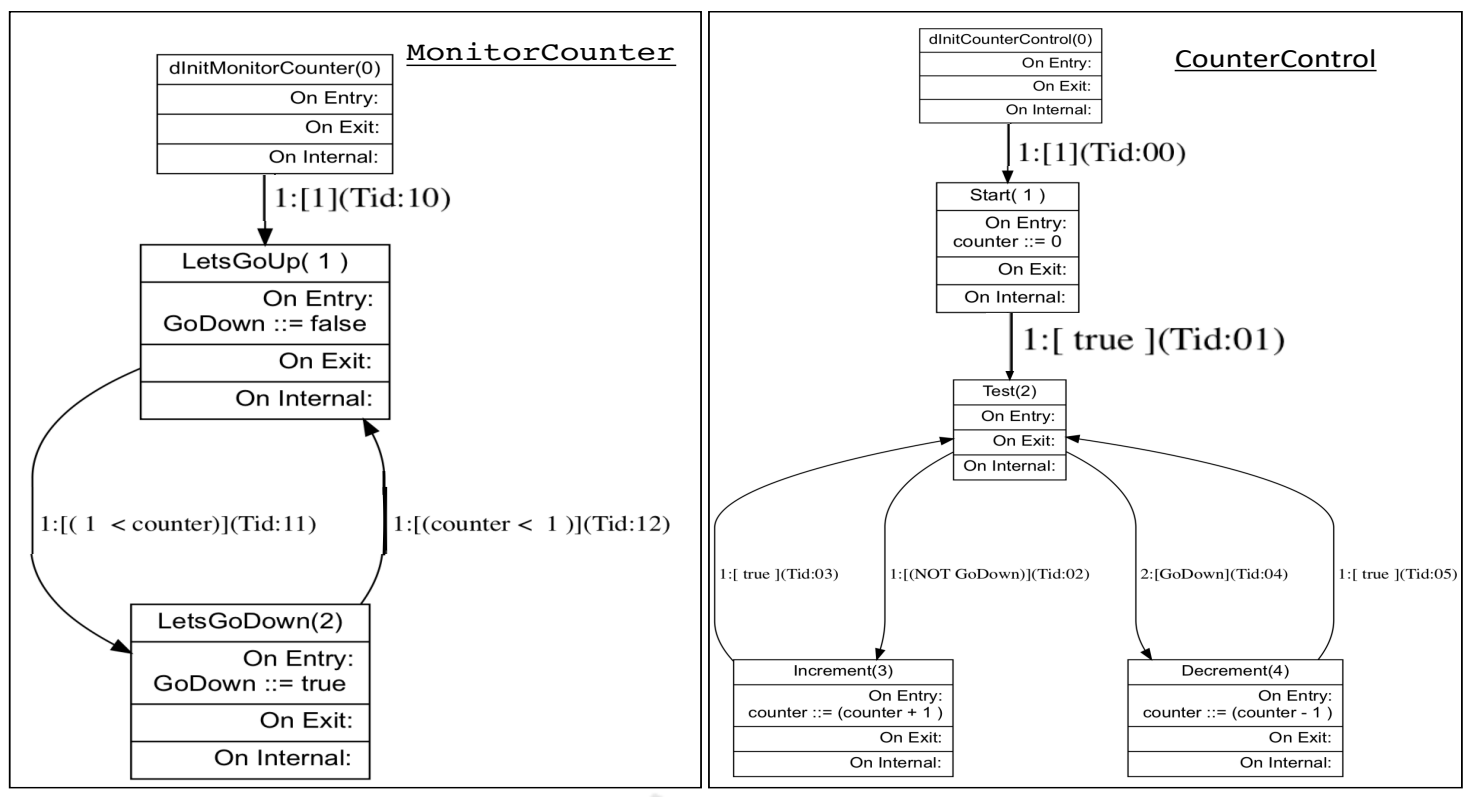

Figure 3: A small illustrative arrangement of two LLFSMs.

variables owned by the module and that are not assigned a value in the code block, we must explicitly indicate that their valuation is not changing.

Illustration: Figure 4 shows the code generated by our ATL implementation of the model-2-model transformation. Line 3 shows that this module owns variable GoDown. It is a whiteboard variable that appears in the LHS of statement in it. Line 5 starts the definition of the conditions for what is the only possible transition that would fire when it is this module's turn. Note that the restrictions in TRANS ensure that a transition fires only if no earlier transition fires. It also shows the effect of the OnEntry of the target state, if the transition fires. We also highlight the statement in Line 25 where our algorithm explicitly indicates that the Boolean variable GoDown is not changing value if it is not this machine's turn.

\subsection{Complexity of the Algorithm}

The construction presented earlier shows that for arrangements of LLFSMs having only blocks of code in the OnEntry sections and with no dependencies, the resulting SMV models have exactly the same semantics as the Ecore arrangements of LLFSMs.

The complexity of the output of the ATL transformation is linear in the total number of states. Observe that there are as many lines in the DEFINE and TRANS sections as there are transitions in the arrangement. For each LLFSM the DEFINE and TRANS have as many lines as transitions in the particular LLFSM. The size of the output (in number of characters) is

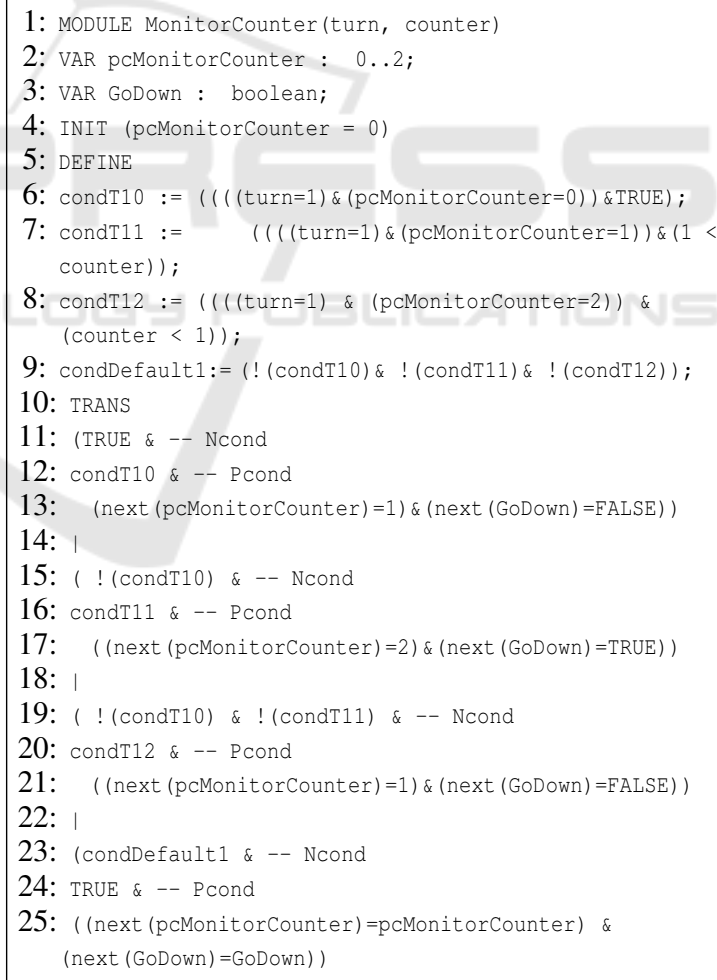

Figure 4: Machine 1 by our ATL m-2-m transformation.

quadratic in the number of transitions of the LLFSM since for the $i$-th transition we must spell out that no previous transition fires. Nevertheless, the reduction in the size of the SMV files is several orders of magnitude the length of the arrangement. For the small 
SMV model of Figure 3, our ATL implementation produces a SMV input file with 110 lines. By contrast, our earlier methods produce an SMV file with 820 lines.

\section{TRANSFORMATION INTO TIMERS}

We now extend the transformation, so that time-value properties can be verified with NuSMV. We will contrast value-domain properties with time-domain properties before we describe our modelling of timers.

\subsection{What are Augmented LLFSMs?}

The subsumption architecture for robotic and embedded systems used LISP-coded LLFSMs (Brooks, 1990; Mataric, 1992). Such LLFSMs were augmented because they use predicates about time. We also discuss the implications of modelling time and constructing Kripke structures for formal verification with implicitly or explicitly time modelling.

The LLFSMs presented in the meta-model of Figure 1 are augmented and can be used for robotic and embedded systems (Estivill-Castro and Hexel, 2018). Such LLFSMs offer Boolean expressions of the form

$$
\operatorname{AFTER}(\langle\text { arithmetic-expression }\rangle) \text {. }
$$

which can be part of the label of any transition. The semantics of the AFTER Boolean expression for a transition $T$ with source state $s_{1}$ and target state $s_{2}$ is that a snapshot $t_{1}$ is taken of the system time when execution arrives at state $s_{1}$ (just before the execution of $s_{1}$ 's OnEntry). At this time, the arithmetic expression that is the argument of the AFTER is evaluated and the resulting value $\Delta$ is also saved. Execution of the ringlet for $s_{1}$ proceeds as normal: the OnEntry is executed and the transitions out of $s_{1}$ are evaluated in the order of the sequence holding them. But when transition $T$ is evaluated, the AFTER is replaced by a comparison of the current system time $t_{2}$ and the value $t_{1}+\Delta$. The AFTER evaluates to true if and only if $t_{2}>t_{1}+\Delta$.

\subsection{Time-domain versus Value-domain}

The original methods for verifying LLFSMs built a Kripke structure (a SMV model) (Estivill-Castro et al., 2012) where the AFTER is analogous to an environment variable. Then, the open model is converted to a closed model as we mentioned earlier: the Kripke structure offers non-deterministic transitions with both valuation (true and false) of the
AFTER. Thus, the verifiable properties are value domain properties. To illustrate this point we reproduce the LLFSMs control of the ubiquitous Microwave Oven (Shlaer and Mellor, 1992; Myers and Dromey, 2009). The Microwave Oven is analogous to the Therac X-ray machine and has been widely discussed in the literature of software engineering and formal verification (Mellor, 2007). Figure 5 reproduces its arrangement of LLFSM. There is a transition for the bell-controller component with source RINGING to BELL_OFF that holds the sound effector active for two units of time after the cooking has finished. There is another transition in the time-controller with an AFTER to decrement the time by 1 unit as long as the door is closed and the button is released.

Value domain properties confirm desirable properties regarding the behaviour of the Microwave. We find particularly illustrative that "After the sound effector is activated, eventually, the sound effector is deactivated" (that is, the bell at the conclusion of cooking does not ring forever). There is a symmetric property that says that if the sound effector is silent and cooking concludes, and the user does not add time for some Kripke states, eventually the sound effector rings. However, because of the construction of the Kripke structure mentioned earlier, there is no way to formulate a property that includes the value $\Delta$ of the argument of an AFTER predicate. The counts of Kripke states are in the sense of bounded NuSMV properties (that is, formulas require a precise number of repetitions of the temporal logic operator X).

\subsection{Transformation of Time Expressions}

We now propose to produce ATL transformations to SMV modules where each appearing AFTER results in a parameterised invocation to one timer module. The timer module explicitly implements the test $t_{2}>t_{1}+\Delta$ as $t_{2}-t_{1}>\Delta$. This means that

- the SMV model only uses integers as large as the highest value for the arithmetic expression that is an argument to an AFTER predicate;

- the integer variables about timers are SMV variables that can appear in CTL or LTL formulas.

Transformation Step: An instance of a timer (a SMV module) interacts with the LLFSM $M$ that labels a transition $T$ with source state $s_{1}$ and target state $s_{2}$ including an AFTER as follows. There are four shared variables between the instance of the timer and the SMV module for $M$. The first three are owned by $M$, and thus parameters to the timer.

1. active: $M$ sets active to true on arrival to $s_{1}$. 


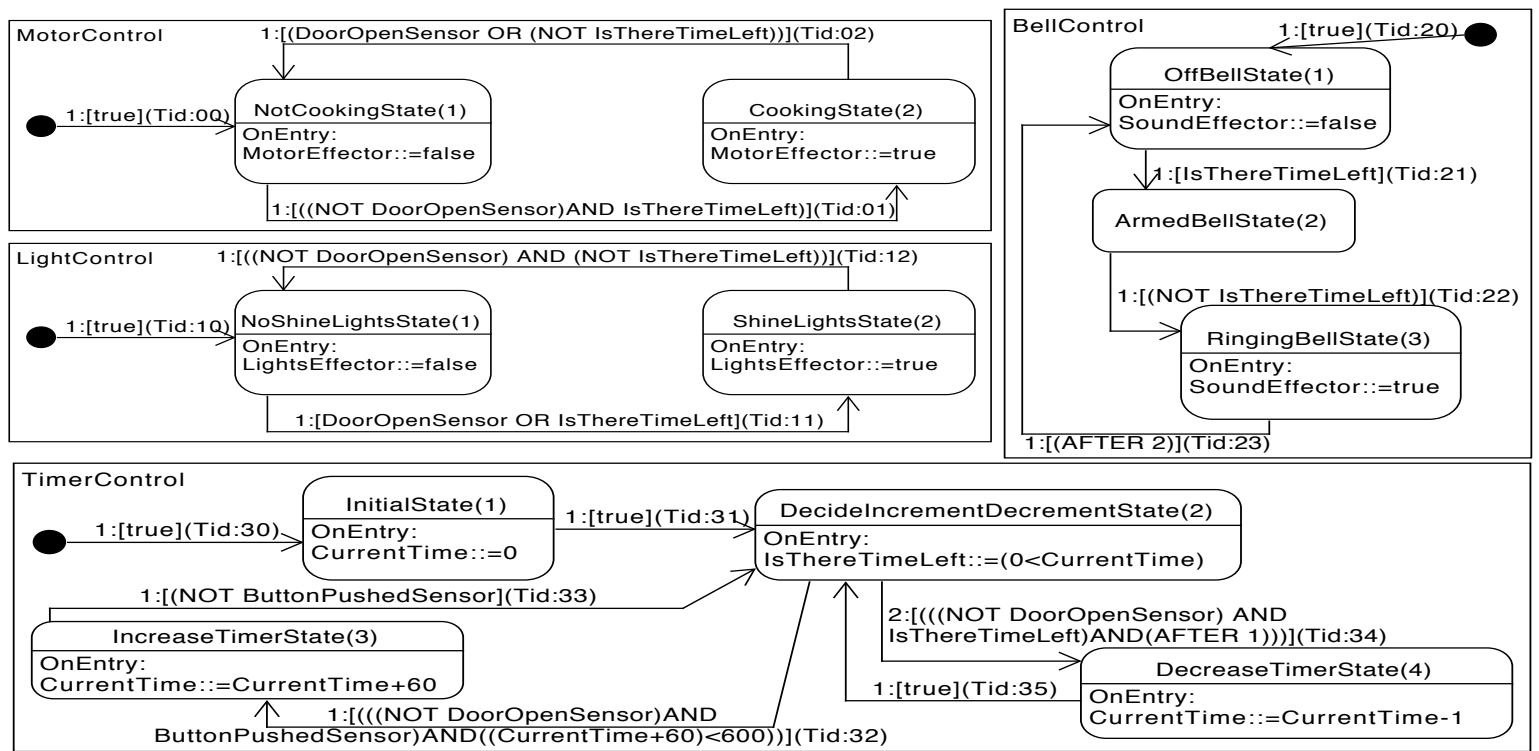

Figure 5: Complete executable and verifiable behavioural model (as LLFSMs) for the requirements (Shlaer and Mellor, 1992; Myers and Dromey, 2009) of the Microwave.

2. bound: $M$ assigns the value of the arithmetic expression to bound also on arrival to the state $s_{1}$.

3. step: Also on entry to $s_{1}, M$ assigns this value as required to decrement the local counter of the timer per turn to the timer.

$M$ makes active false in every target state of $s_{1}$ (not only $s_{2}$ ).

Illustration: For example, in the time-control of the microwave, the user may push the button and add even more time to a microwave already counting down. In that case, the LLFSM moves to add time when button_pushed is true and abandons the waiting for a second to pass to decrement the current time.

Transformation Step: The variable owned by the instance of the timer is finished. This is set to false at the start, but becomes true when the timer finds its local time counter (which started at zero when activated) reaches bound. The variable finished replaces the predicate AFTER in the transition $T$.

Illustration: This model-2-model ATL transformation is illustrated in Figure 6. It is non-trivial as it must be performed for each transition that holds one or more AFTER predicates in the Boolean expression that it labels them. Therefore, the set of variables for each of these predicates are tagged by the machine number, the Tid of the transition.

Transformation Step: An instance of the timer module is created in the main module of the SMV model. Only one generic timer module, a LLFSM itself, is defined. But, as many instances as AFTER predicates in the arrangement are constructed. They become part of the SMV model and properties regard-

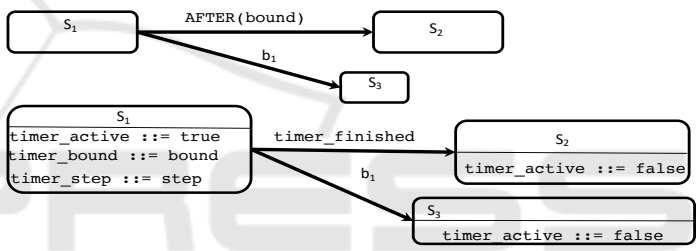

Figure 6: The transformation that replaces LLFSMs augmented with AFTER-predicates to LLFSMs.

ing the behaviour of the arrangement and the bounds in its AFTER predicates can be verified.

Illustration: Figure 7 shows the generic timer as an LLFSM which we can produce in our LISP execution of LLFSMs for validation.

Transformation Step: The SMV module for the only generic timer can be obtained by applying the ATL transformation described in the previous section. However, the ATL-transformation not only performs the replacement of all the AFTER-predicates, but also delivers one SMV generic timer module and the construction of its instances in main.

Illustration: Figure 8 shows the snippet NuSMV code in the main module resulting from the ATL program applied to the executable model from Figure 5.

\subsection{Complexity and Efficiency}

Our ATL transformation delivers also a succinct SMV when the executable LLFSM arrangement has AFTERpredicates. The number of modules is the number of LLFSMs in the arrangement plus one. The total num- 


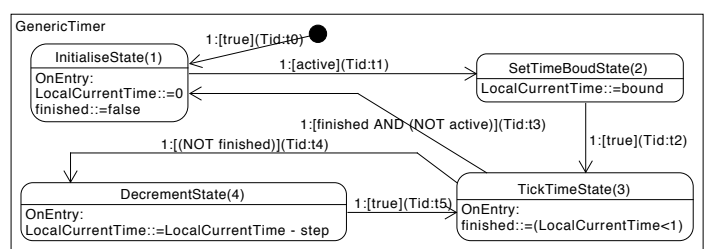

Figure 7: The generic timer is also a LLFSM.

ber of instances of modules is the number of LLFSMs in the arrangement plus the number of occurrences of AFTER-predicates in the arrangement.

Using a desktop computer with an Intel quad-core processor, 32 GB of RAM, and MacOS, it is possible to verify four properties that express requirements of the microwave, in $551 \mathrm{~s}, 553 \mathrm{~s}, 853 \mathrm{~s}$, and $853 \mathrm{~s}$, respectively, over NuSMV models generated by our earlier algorithms (see the introduction). In contrast, over the NuSMV model generated by the algorithm of section 3, the same properties can be verified in 29 s, 32 s, 43 s, and 43 s, respectively, using a MacOS laptop with an Intel i7 processor and $16 \mathrm{~GB}$ of RAM. We must highlight that, with some CTL properties, our earlier algorithms did not even finish after running for one hour on the laptop.

\subsection{Discussion}

Time-domain properties are now expressible for SMV directly. For example, SMV properties such as

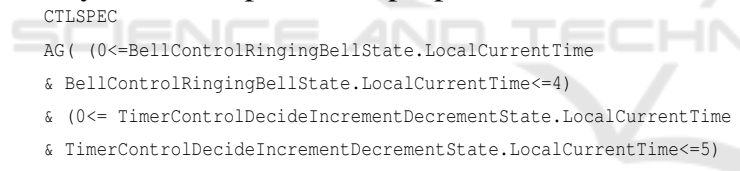

that checks that the variable LocalcurrentTime, of each timer instance, stays within respective bounds is true (and the time required is minimal, because its verification is tested by the compilation of the model matching the bounds of the variable definitions).

Similarly, we can now revisit the discussion of Section 4.2. As opposed to Kripke structures generated by closing the system when time predicates appear in the transition, now we can check specific realtime properties.

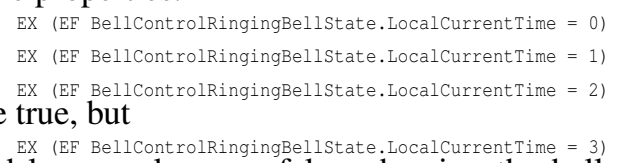
and larger values are false, showing the bell would never ring for more than two time units.

The transformations discussed in Section 3 are not necessarily completely equivalent regarding time units in the micro-scale at which LLFSMs run. These transformations (breaking the sections of a state into separate states, for example) impact the predefined schedule. The machine in question would need several turns to complete for what was earlier a single turn. Nevertheless, our methods provide the advantage that such expansion of a few turns to one LLFSM in the arrangement are conditions that should also verified. Any arrangement of LLFSMs (executable model of behaviour) that relies on specific timing from subsections of states of participant LLFSMs for fairness, liveliness or deadlock-free properties, is a fragile behaviour design. Similarly, if the correctness of the arrangement depends on the order of LLFSMs in the arrangement, we will have a fragile design. Verifying these weaknesses in designs is now possible. The efficient Kripke structures we are capable of producing with the algorithms here enable verification which previously was infeasible.

\section{CONCLUSIONS}

We have presented an algorithm (that is implemented as an ATL model-2-model transformation) that achieves succinct SMV models from generic executable forms of arrangements of LLFSMs. Moreover, this transformation enables involving time predicates and later verifying properties about them, thus extending the types of properties from the value domain to the time domain.

\section{REFERENCES}

Besnard, V., Brun, M., Jouault, F., Teodorov, C., and Dhaussy, P. (2018). Unified LTL verification and embedded execution of UML models. 21th ACM/IEEE Int. Conf. Model Driven Engineering Languages and Systems, MODELS '18, p. 112-122, NY, USA. ACM.

Bhaduri, P. and Ramesh, S. (2004). Model checking of statechart models: Survey and research directions.

Biere, A., Cimatti, A., Clarke, E., and Zhu, Y. (1999). Symbolic model checking without BDDs. Tools and Algorithms for the Construction and Analysis of Systems, p. 193-207. Springer Berlin Heidelberg.

Brooks, R. (1990). The behavior language; user's guide. Tech. Rpt. AIM-1227, MIT, Dept. Electronics and CS.

Cheng, K.-T. and Krishnakumar, A. S. (1993). Automatic functional test generation using the extended finite state machine model. 30th Int. Design Automation Conference, DAC '93, p. 86-91, NY, USA. ACM.

Cimatti, A., Clarke, E., Giunchiglia, F., and Roveri, M. (2000). NuSMV: a new symbolic model checker. Int. J. Software Tools for Technology Transfer, 2(4):410 425 .

Clarke, E. and Heinle, W. (2000). Modular translation of statecharts to SMV. Tech. Rpt., School of Computer Science, Carnegie Mellon U., Pittsburg, PA. 
VAR

OvenMotorControl: OvenMotorControl (turn, DooropenSensor, OvenTimerControl.IsThereTimeLeft);

OvenLightControl: OvenLightControl (turn, DooropenSensor, OvenTimerControl. IsThereTimeLeft)

ovenBellControl: OvenBellControl (turn, OvenTimerControl.IsThereTimeLeft, TimerOvenBellControlRingingBellstate.finished);

ovenTimerControl: OvenTimerControl (turn DooropenSensor, ButtonPushedSensor, TimerovenTimerControlDecideIncrementDecrementState.finished);

TimerovenBellControlRingingBellstate: Timer(turn, 4, 2, 1, OvenBellControl.OvenBellControl_RingingBellstate_active);

TimerovenTimerControlDecideIncrementDecrementState: Timer(turn, 5, 1, 1, ovenTimerControl.OvenTimerControl_DecideIncrementDecrementState_active);

Figure 8: The instantiation of modules in main for the SMV model of the executable model of Figure 5.

Devadas, S., Keutzer, K., and Krishnakumar, A. S. (1991). Design verification and reachability analysis using algebraic manipulation. IEEE Int. Conf. Computer Design on VLSI in Computer \&Amp; Processors, ICCD '91, p. 250-258, IEEE Computer Soc.

Drusinsky, D. (2006). Modeling and Verification Using UML Statecharts: A Working Guide to Reactive System Design, Runtime Monitoring and Executionbased Model Checking. Newnes, Newton, MA, USA.

Estivill-Castro, V. and Hexel, R. (2013). Module isolation for efficient model checking and its application to FMEA in model-driven engineering. ENASE 8th Int. Conf. on Evaluation of Novel Approaches to Software Engineering, p. 218-225. SciTePress.

Estivill-Castro, V. and Hexel, R. (2018). Verifiable parameterised behaviour models - for robotic and embedded systems. 6th Int. Conf. on Model-Driven Engineering and Software Development, MODELSWARD, p. 364371. SciTePress.

Estivill-Castro, V. and Hexel, R. (2019). Resolving the asymmetry of on-exit versus on-entry in executable models of behaviour. 7th Int. Conf. Model-Driven Engineering and Software Development, MODELSWARD, p. 49-61. SciTePress.

Estivill-Castro, V., Hexel, R., and Ramirez Regalado, A. (2016). Architecture for logic programing with arrangements of finite-state machines. 1st CPSWeek Workshop on Declarative Cyber-Physical Systems, $D C P S$, p. 1-8. IEEE Computer Soc..

Estivill-Castro, V., Hexel, R., and Rosenblueth, D. A. (2012). Efficient modelling of embedded software systems and their formal verification. 19th AsiaPacific Software Engineering Conference, APSEC 2012, p. 428-433. IEEE.

Estivill-Castro, V. and Rosenblueth, D. A. (2011). Model checking of transition-labeled finite-state machines. Software Engineering, Business Continuity, and Education - Int. Conf. ASEA, p. 61-73. Springer.

Gansner, E. R., Koutsofios, E., and North, S. (2015). Drawing graphs with dot.

Harel, D., Pnueli, A., Lachover, H., Naamad, A., Politi, M., Sherman, R., Shtull-Trauring, A., and Trakhtenbrot, M. (1990). Statemate: A working environment for the development of complex reactive systems. IEEE Trans. Softw. Eng., 16(4):403-414.

ITU-T Study Group 17 (2002). Formal description techniques (FDT) - Specification and Description Language $(S D L)$.

Kang, I. and Lee, I. (1993). A state minimization algorithm for communicating state machines with arbitrary data space. Tech. Rpt MS-CIS-93-07, Dpt. of Computer \& Information Science, U. of Pennsylvania.
Lamport, L. (1984). Using time instead of timeout for faulttolerant distributed systems. ACM T. on Programming Languages and Systems, 6:254-280.

Mataric, M. (1992). Integration of representation into goaldriven behavior-based robots. IEEE T. Robotics and Automation, 8(3):304 -312.

McColl, C. and Estivill-Castro, V. Hexel, R. (2017). An $\mathrm{OO}$ and functional framework for versatile semantics of logic-labelled finite state machines. ICSEA : 12th Int. Conf. on Software Engineering Advances, p. 238243. IARIA, Curran.

McMillan, K. L. (1992). Symbolic Model Checking - An approach to the state explosion problem. $\mathrm{PhD}$ thesis, Carnegie Mellon U., Pittsburgh, CMU-CS-92-131.

Meenakshi, B., Bhatnagar, A., and Roy, S. (2006). Tool for translating Simulink models into input language of a model checker. Formal Methods and Software Engineering, p. 606-620, . Springer Berlin Heidelberg.

Mellor, S. J. (2000). UML point/counterpoint: Modeling complex behavior simply. Embedded Systems Programming.

Mellor, S. J. (2007). Embedded systems in UML. OMG White paper. www.omg.org/news/whitepapers/ label: We can generate Systems Today.

Myers, T. and Dromey, R. G. (2009). From requirements to embedded software - formalising the key steps. 20th Australian Software Engineering Conf. (ASWEC), p. 23-33, Gold Cost, Australia. IEEE Computer Soc.

Ozik, J., Collier, N., Combs, T., Macal, C. M., and North, M. (2015). Repast simphony statecharts. J. Artificial Societies and Social Simulation, 18(3):11.

Poledna, S. (1996). Fault-Tolerant Real-Time Systems: The Problem of Replica Determinism. Kluwer, MA, USA.

Rumbaugh, J., Blaha, M., Premerlani, W., Eddy, F., and Lorensen, W. (1991). Object-oriented Modeling and Design. Prentice-Hall, NJ, USA.

Samek, M. (2008). Practical UML Statecharts in $\mathrm{C} / \mathrm{C}++$, Second Edition: Event-Driven Programming for Embedded Systems. Newnes, MA, USA.

Selic, B., Gullekson, G., and Ward, P. T. (1994). Real-time Object-oriented Modeling. Wiley, NY, USA.

Seshia, S. A., Sharygina, N., and Tripakis, S. (2018). Modeling for verification. , Handbook of Model Checking, p. 1-26, Cham. Springer.

Shlaer, S. and Mellor, S. J. (1992). Object lifecycles: modeling the world in states. Yourdon P., N.J.

von der Beeck, M. (1994). A comparison of statecharts variants. 3rd Int. Symposium Organized Jointly with the Working Group Provably Correct Systems on Formal Techniques in Real-Time and Fault-Tolerant Systems, ProCoS, p. 128-148, Berlin. Springer.

Winskel, G. (1993). The Formal Semantics of Programming Languages: An Introduction. MIT, Cambridge, MA. 